should this time be too short to alter the NMR phase by quadrupole coupling, the probability that the event will contribute to line-broadening is one-half.

The reaction rate is perhaps within an order of magnitude of the collision frequency. This implies that the activation energy must be quite small and that large angles of attack are nevertheless favorable to reaction.
Preliminary experiments with sodium iodide indicate a still higher rate. Effects of ionic strength and ionic hydration are being investigated.

There is a wide variety of reaction systems amenable to similar studies, so that the application of quadrupole broadening of nuclear magnetic resonance lines should become a powerful technique in chemical kinetic studies.

\title{
Raman Spectroscopy in Liquid Ammonia Solutions. Vibrational Frequencies and Force Constants for Isotopic Species of the Borohydride Ion Having Tetrahedral Symmetry* $\dagger$
}

\author{
Allan R. Emery and Robert C. Taylor \\ Department of Chemistry, University of Michigan, Ann Arbor, Michigan
}

(Received January 28, 1958)

\begin{abstract}
All fundamentals of the ions ${ }^{11} \mathrm{BH}_{4}^{-},{ }^{11} \mathrm{BD}_{4}^{-},{ }^{10} \mathrm{BH}_{4}^{-}$, and ${ }^{10} \mathrm{BD}_{4}^{-}$have been observed in the Raman spectra of some alkali metal salts dissolved in liquid $\mathrm{NH}_{3}$ or $\mathrm{ND}_{3}$ at $-50^{\circ} \mathrm{C}$. A complete assignment of all observed frequencies has been made and force constants have been calculated by the $F G$ method of Wilson.
\end{abstract}

\section{INTRODUCTION}

$\mathbf{I}^{\mathrm{N}}$ $\mathrm{N}$ a previous article ${ }^{1}$ the Raman spectra and assignments of the ${ }^{11} \mathrm{BH}_{4}^{-}$and ${ }^{11} \mathrm{BD}_{4}^{-}$ions dissolved in liquid ammonia were reported. However, solvent interference prevented the observation of one fundamental of the borohydride ion and confused the interpretation of the $B-D$ stretching region of the borodeuteride spectrum. Since this ion is one of the simplest boron hydride structures, it is desirable to have a complete and unequivocal assignment of fundamentals, and consequently the work has been extended making further use of isotopic substitution for both hydrogen and boron. In particular, employment of the isotopic solvent, $\mathrm{ND}_{3}$, has successfully eliminated interference by solvent bands and has made possible the observation of all the fundamentals of the various isotopically substituted ions. The use of ammonia as a solvent was dictated by solubility considerations, the fact that hydrolysis occurs in aqueous solution and the desirability of a simple solvent spectrum. Since chemical exchange of hydrogen between the borohydride ion and ammonia occurs extremely slowly, if at all, the two isotopic solvents could be used interchangeably depending on the spectral region of interest.

The final assignment which has been made agrees with all available data and confirms the tetrahedral

* Based on a dissertation submitted by Allan R. Emery in partial fulfillment of the requirements of the Ph.D. degree to the Horace H. Rackham School of Graduate Studies, University of Michigan, June, 1957.

+ Presented at the Symposium on Molecular Structure and Spectroscopy, Columbus, Ohio, June, 1957.

1 Taylor, Schultz, and Emery, J. Am. Chem. Soc. 80, 27 (1958). structure of the ion. The values of the fundamentals have been used to evaluate the force constants of a complete valence force potential function.

\section{EXPERIMENTAL}

Sodium and lithium borohydrides containing boron-11 in its natural abundance of $81 \%$ were obtained from Metal Hydrides, Inc., and purified before use by removing all ammonia insoluble material. Potassium borohydride was prepared by metathesis between $\mathrm{NaBH}_{4}$ and $\mathrm{KOH}$ according to a recently described procedure. ${ }^{2}$ Borodeuterides and all compounds containing boron-10 were prepared by one of two procedures depending on the alkali metal. Lithium compounds were obtained by reacting an ether slurry of lithium hydride or deuteride with the appropriate diborane in a sealed reaction tube. Sodium and potassium salts were prepared by the reaction of the appropriate diborane with sodium or potassium tetramethoxyborate. The reactions involved have been reported by Schlesinger et al. ${ }^{3}$ All compounds were purified to remove ammonia insoluble material before use. Boron-10 was obtained in $96 \%$ enrichment as the calcium fluoride-boron trifluoride complex from Union Carbide and Carbon Corporation, Oak Ridge, Tennessee. The $\mathrm{BF}_{3}$ was converted to diborane by reaction of its etherate with lithium aluminum hydride or deuteride. ${ }^{4}$ Although a small amount of hydrogen was intro-

\footnotetext{
2 Banus, Bragdon, and Hinckley, J. Am. Chem. Soc. 76, 3848 (1954).

3 Schlesinger et al. J. Am. Chem. Soc. 75, 186-224 (1953).

4 Shapiro, Weiss, Schmich, Skolnik, and Smith, J. Am. Chem. Soc. 74, 901 (1952).
} 
duced in the synthesis of the deuterated compounds, its amount was estimated at not more than $2-3 \%$ of the total deuterium present and no difficulty was met in the interpretation of the spectra because of the presence of a small percentage of isotopically mixed ions. Deuterated ammonia was prepared in a small steel cylinder by the reaction of $\mathrm{D}_{2} \mathrm{O}$ with freshly prepared $\mathrm{Mg}_{3} \mathrm{~N}_{2}$. Its spectrum showed insignificant amounts of hydrogen to be present.

The preparation of the samples has been described previously. ${ }^{1}$ In most cases they consisted of 1-2 $\mathrm{ml}$ of solution whose concentration lay in the range between 2-4 molar. No significant changes of the spectra with concentration were noted. Reference to the spectroscopic equipment has been given in the earlier paper. ${ }^{1}$

\section{EXPERIMENTAL RESULTS AND DISCUSSION}

The observed frequencies, assignments, and polarization characteristics of the bands of the alkali metal borohydrides in ammonia solution are listed in Table I

TABLE I. Vibrational frequencies and assignments for the borohydride ion in liquid ammonia solution (in $\mathrm{cm}^{-1}$ ).

\begin{tabular}{lclllc}
\hline \hline $\mathrm{Li}^{11 \mathrm{BH}}$ & $\mathrm{Na}_{4}{ }^{11} \mathrm{BH}_{4}$ & $\mathrm{~K}^{11} \mathrm{BH}_{4}$ & $\mathrm{Li}^{10} \mathrm{BH}_{4}$ & $\mathrm{Na}^{10} \mathrm{BH}_{4}$ & $\begin{array}{c}\text { Assign- } \\
\text { ments }\end{array}$ \\
\hline $1082 \pm 2^{\mathrm{a}}$ & $1080 \pm 2$ & $1084 \pm 2$ & $1085 \pm 2$ & $1093 \pm 2$ & $\nu_{4}$ \\
$1202 \pm 3$ & $1210 \pm 3$ & $1213 \pm 3$ & $1206 \pm 5$ & $1208 \pm 3$ & $\nu_{2}$ \\
$2146 \pm 5$ & $2146 \pm 3$ & $2154 \pm 3$ & $2150 \pm 3$ & $2161 \pm 3$ & $2 \nu_{4}$ \\
$2265 \pm 2$ & $2264 \pm 2$ & $2266 \pm 3$ & $2268 \pm 2$ & $2270 \pm 2$ & $\nu_{1}$ \\
$2402 \pm 3$ & $2398 \pm 3$ & $2404 \pm 3$ & $2409 \pm 5$ & $2405 \pm 5$ & $2 \nu_{2}$ \\
\hline
\end{tabular}

astimated standard deviations.

while the corresponding data for the alkali metal borodeuterides are given in Table II. The values tabulated are averages obtained from several plates, no distinction having been made between $\mathrm{NH}_{3}$ and $\mathrm{ND}_{3}$ solutions. $\neq \mathrm{A}$ small but real shift in band position with cation was apparent and data accordingly have been given for each salt. Data obtained from the sodium salt have been used for purposes of the discussion and in the force constant calculations, since they form the most complete set and are roughly intermediate between the values for the other two alkali metals. Some of the previously published frequencies of the boron-11 compounds ${ }^{1}$ have been revised on the basis of a larger number of better quality spectra; these changes are largely within the experimental uncertainty of the previous values.

Satisfactory assignments have been made assuming a

\footnotetext{
$\ddagger$ Evidence suggesting small systematic differences in band position between the two solvents was obtained in the case of one or two of the fundamentals. The specific nature of solute-solvent interaction which this suggests is supported by the observation that the maximum of the symmetrical stretching frequency of $\mathrm{BH}_{4}^{-}$differs by about $25 \mathrm{~cm}^{-1}$ bet ween $\mathrm{NH}_{3}$ and $\mathrm{H}_{2} \mathrm{O}$ as solvents, and by the fact that the alkali metal borohydrides form stable ammoniates. For most of the bands, however, the differences, if real, between $\mathrm{NH}_{3}$ and $\mathrm{ND}_{3}$ solutions were less than the associated probable error in the band positions and have not been considered significant.
}

TABLE II. Vibrational frequencies and assignments for the borodeuteride ion in liquid ammonia and deuteroammonia solutions (in $\mathrm{cm}^{-1}$ ).

\begin{tabular}{rrrrrl}
\hline $\mathrm{Li}^{11} \mathrm{BD}_{4}$ & $\mathrm{Na}^{11} \mathrm{BD}_{4}$ & \multicolumn{1}{c}{$\mathrm{K}^{11} \mathrm{BD}_{4}$} & \multicolumn{1}{c}{$\mathrm{Li}^{1{ }^{10} \mathrm{BD}}$} & \multicolumn{1}{c}{$\mathrm{Na}^{10} \mathrm{BD}_{4}$} & $\begin{array}{c}\text { Assign- } \\
\text { ments }\end{array}$ \\
\hline $822 \pm 2^{\mathrm{B}}$ & $823 \pm 2$ & $827 \pm 2$ & $833 \pm 3$ & $834 \pm 2$ & $\nu_{4}$ \\
$854 \pm 2$ & $855 \pm 2$ & $864 \pm 3$ & & $858 \pm 4$ & $\nu_{2}$ \\
$1569 \pm 3$ & $1570 \pm 3$ & $1572 \pm 3$ & $1570 \pm 2$ & $1571 \pm 2$ & $\nu_{1}$ \\
$1636 \pm 4$ & $1636 \pm 4$ & $1636 \pm 4$ & $1645 \pm 3$ & $1650 \pm 5$ & $2 \nu_{4}(E$ \\
& & & & & or $\left.F_{2}\right)$ \\
$1668 \pm 3$ & $1668 \pm 3$ & $1670 \pm 5$ & $1683 \pm 3$ & $1680 \pm 2$ & $2 \nu_{4}\left(A_{1}\right)$ \\
$1698 \pm 3$ & $1696 \pm 5$ & $1694 \pm 3$ & $1704 \pm 3$ & $1706 \pm 4$ & $\nu_{3}$ \\
$2240 \pm 3$ & $2238 \pm 4$ & $2238 \pm 5$ & $2243 \pm 3$ & $2245 \pm 2$ & $\mathrm{BD}_{3} \mathrm{H}^{-}$ \\
& & & & & ion \\
\hline
\end{tabular}

astimated standard deviations.

tetrahedral configuration for the ion belonging to the $T_{d}$ point group. This model predicts one $A_{1}$, one $E$, and two $F_{2}$ fundamentals, all Raman active. Isotopic substitution of deuterium for hydrogen should result in a marked shift in all frequencies while isotopic substitution of the central boron atom should not affect the $A_{1}$ and $E$ frequencies but shift the $F_{2}$ fundamentals by a small amount.

The $A_{1}$ stretching frequency is easily identified as the $2264 \mathrm{~cm}^{-1}$ band in the hydrogen compounds and the $1570 \mathrm{~cm}^{-1}$ band in the deuterium from intensity and polarization characteristics. A shift in the apparent position of this fundamental in the borohydride ion on substitution of ${ }^{10} \mathrm{~B}$ for ${ }^{11} \mathrm{~B}$ probably is due to the fact that $\nu_{1}$ is superimposed on $\nu_{3}$ making the observed band maximum sensitive to shifts in the latter. In the borodeuteride ion, a Fermi resonance occurs between $\nu_{1}$ and the overtone of the band at $823 \mathrm{~cm}^{-1}$ which shifts the fundamental some $30 \mathrm{~cm}^{-1}$ from its unperturbed value.

The assignment of $\nu_{2}$, the doubly degenerate bending vibration, to the band at $1210 \mathrm{~cm}^{-1}$ in the hydrogen ion and $855 \mathrm{~cm}^{-1}$ in the deuterium is confirmed in the present work by the absence of a ${ }^{10} \mathrm{~B}$ isotope shift. The other bending fundamental, the triply degenerate $\nu_{4}$, was not observed previously because of interference by a solvent band; its position was inferred to be near $1080 \mathrm{~cm}^{-1}$ from the assignment of a band near 2150 $\mathrm{cm}^{-1}$ as its first overtone. When $\mathrm{ND}_{3}$ is used in place of $\mathrm{NH}_{3}$, no solvent bands occur from about 900 to $1175 \mathrm{~cm}^{-1}$. The spectrum of $\mathrm{BH}_{4}^{-}$in this solvent showed the expected fundamental as a band of moderately low intensity with a maximum at $1080 \mathrm{~cm}^{-1}$. The corresponding band of $\mathrm{BD}_{4}^{-}$appeared at $823 \mathrm{~cm}^{-1}$. Confirmation of the assignment was obtained from the ${ }^{10} \mathrm{~B}$ isotope shifts of 13 and $11 \mathrm{~cm}^{-1}$ respectively.

The remaining triply degenerate fundamental, $\nu_{3}$, was the most difficult of the four to identify because of near superposition by $\nu_{1}$ or overtones in both the hydrogen and deuterium cases. In Fig. 1, $\nu_{3}$, appears as a shoulder on the low-frequency side of the 2264 $\mathrm{cm}^{-1}$ band of the $\mathrm{BH}_{4}-$ ion. Confirmation of its presence and a better measurement of its position was obtained by polarizing the incident light so that the 


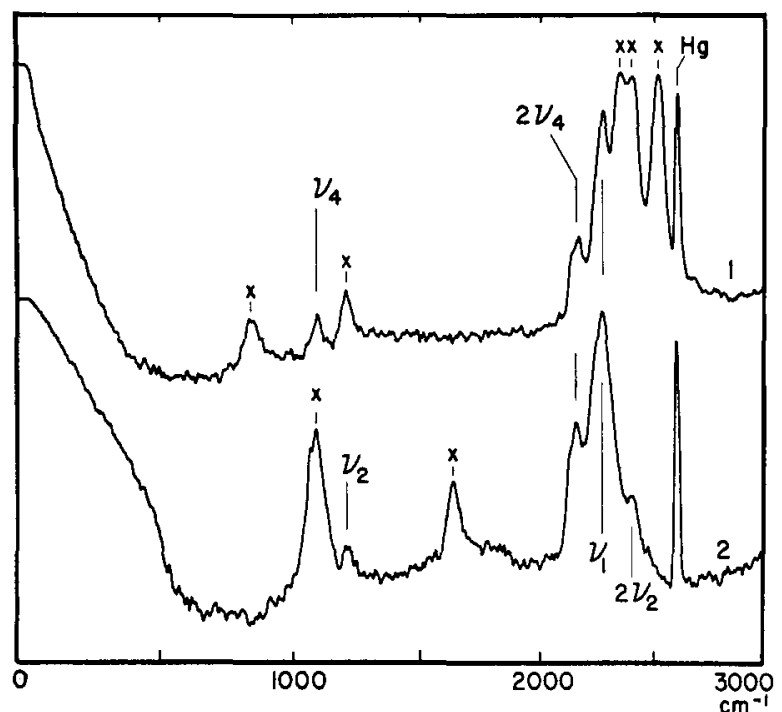

Fig. 1. Raman spectrum of $\mathrm{Na}^{10} \mathrm{BH}_{4}$ dissolved in liquid $\mathrm{ND}_{3}(1)$ or liquid $\mathrm{NH}_{3}(2)$. Solvent bands indicated by $(\mathrm{x})$.

intensity of $\nu_{1}$ was greatly reduced. In theory, the $A_{1}$ mode of a tetrahedral $\mathrm{XY}_{4}$ molecule is completely polarized in the Raman effect but because of convergence error in the incident light, this is difficult to demonstrate. In Fig. 2, a spectrum taken with the electric vector of the incident light polarized parallel to the observation direction shows $\nu_{3}$ as a weak but distinct maximum. Solvent interference necessitated the use of $\mathrm{ND}_{3}$ for the $\mathrm{BD}_{4}^{-}$ion, and again a satisfactory assignment of the four bands observed in the B-D stretching region could not be attained without the help of polarized spectra. The two polarized bands shown in Fig. 3 at 1570 and $1668 \mathrm{~cm}^{-1}$ are assigned to $\nu_{1}$ and the $A_{1}$ component of $2 \nu_{4}$, respectively. They both have been shifted from their expected positions by Fermi resonance. Of the other two bands at 1636 and $1696 \mathrm{~cm}^{-1}$, the latter is assigned to $\nu_{3}$ since it gives better agreement with the product rule and is more intense. It shifts $10 \mathrm{~cm}^{-1}$ on ${ }^{10} \mathrm{~B}^{-}$substitution. The

FIG. 2. The B-H stretching region in the Raman spectrum of ${ }^{11} \mathrm{BH}_{4}{ }^{-} .1$, perpendicularly polarized incident light; 2 , parallel polarized incident light.

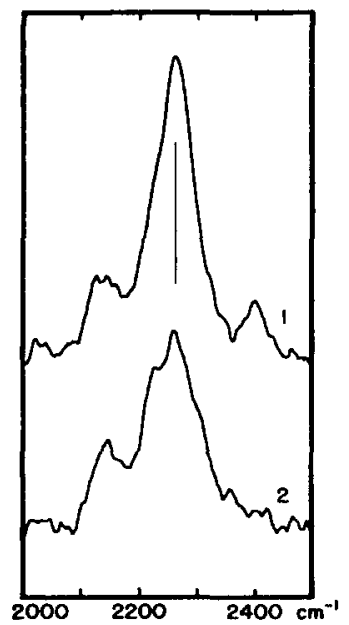

apparent position and breadth of this band may have been influenced by some contribution from $2 \nu_{2}$ which falls at approximately this position but does not have the correct symmetry to resonate. The remaining band at $1636 \mathrm{~cm}^{-1}$ is assigned to the $E$ and/or $F_{2}$ components of $2 \nu_{4}$, the $F_{2}$ component perhaps being enhanced in intensity by resonance with $\nu_{3}$. It is also possible that this band may involve a $\mathrm{B}-\mathrm{D}$ stretching motion of the $\mathrm{BD}_{3} \mathrm{H}^{-}$species which was present in small amounts.

Comparison of the observed frequency product ratios of the four isotopic ions with those predicted

FIG. 3. The B-D stretching region in the Raman spectrum of ${ }^{11} \mathrm{BD}_{4}^{-}$ dissolved in liquid $\mathrm{ND}_{3}$. 1 , perpendicularly polarized incident light; 2 , parallel polarized incident light.

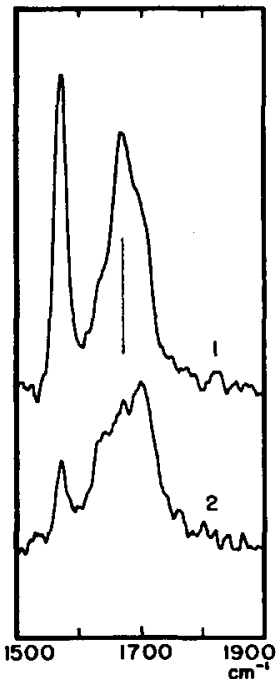

TARLE III. Application of the Teller-Redlich product rule to the borohydride ion.

\begin{tabular}{rcccc}
\hline Ratio & Class & $\begin{array}{c}\text { Frequency } \\
\text { theoret. }\end{array}$ & $\begin{array}{c}\text { Product ratio } \\
\text { observed }\end{array}$ & Dev. \\
\hline${ }^{11} \mathrm{BD}_{4}^{-}$ & $A_{1}$ & 1.414 & 1.443 & $+2.1 \%$ \\
${ }^{11} \mathrm{BH}_{4}^{-}$ & $E$ & 1.414 & 1.414 & 0 \\
& $F_{2}$ & 1.776 & 1.736 & -2.3 \\
${ }^{10} \mathrm{BD}_{4}^{-}$ & $A_{1}$ & 1.414 & 1.445 & +2.2 \\
${ }^{10} \mathrm{BH}_{4}^{-}$ & $E$ & 1.414 & 1.408 & -0.4 \\
& $F_{2}$ & 1.761 & 1.727 & -1.9
\end{tabular}

Sum difference: $\left.\sum\left[\nu_{i}{ }^{2}{ }^{10} \mathrm{BH}_{4}{ }^{-}\right)-\nu_{i}{ }^{2}\left({ }^{11} \mathrm{BH}_{4}{ }^{-}\right)\right]=5.52 \times 10^{4}$ $\left(F_{2}\right.$ class $) \quad \Sigma\left[\nu_{i}^{2}\left({ }^{10} \mathrm{BD}_{4}^{-}\right)-\nu_{i}^{2}\left(11 \mathrm{BD}_{4}^{-}\right)\right]=5.23 \times 10^{4}$

by the product rule is shown in Table III. The agreement in general is satisfactory with the exception of the $A_{1}$ class where some of the fundamentals are shifted by resonance. The normal effect of anharmonicity is to cause the observed product ratios as written to be somewhat less than the theoretical. Also included in Table III are data for the isotopic sum rule applied to the $F_{2}$ class frequencies.

\section{NORMAL COORDINATE TREATMENT}

The vibrational analysis was carried out by means of symmetry factored $F$ and $G$ matrices as described by Wilson. ${ }^{5}$ Specific details of the application of this

${ }^{5}$ Wilson, Decius, and Cross, Molecular Vibrations (McGrawHill Book Company, Inc., New York, 1955). 
TABLE IV. Comparison of observed and calculated fundamental vibrational frequencies of the various isotopic species of the borohydride ion.

\begin{tabular}{|c|c|c|c|c|c|c|c|c|c|c|c|c|c|}
\hline & & \multicolumn{3}{|c|}{${ }^{11} \mathrm{BH}_{4}^{-}$} & \multicolumn{3}{|c|}{${ }_{10} \mathrm{BH}_{4}^{-}$} & \multicolumn{3}{|c|}{${ }^{1 B^{\prime} D_{4}^{-}}$} & \multicolumn{3}{|c|}{${ }^{10} \mathrm{BD}_{4}^{-}$} \\
\hline \multicolumn{2}{|c|}{ Fundamental } & Obs & Calc & Dev & Obs & Calc & Dev & Obs & Calc & Dev & Obs & Calc & Dev \\
\hline$A_{1}$ & $\nu_{1}$ & 2264 & 2267 & +0.1 & 2270 & 2267 & -0.1 & $(1570)^{a}$ & 1604 & & $(1571)$ & 1604 & \\
\hline$E$ & $\nu_{2}$ & 1210 & 1210 & 0 & 1208 & 1210 & +0.2 & 855 & 856 & +0.1 & 858 & 856 & -0.2 \\
\hline$F_{2}$ & $\nu_{3}$ & 2244 & 2252 & +0.4 & 2250 & 2265 & +0.7 & 1696 & 1688 & -0.5 & 1706 & 1707 & +0.1 \\
\hline & $\nu_{4}$ & 1080 & 1089 & +0.9 & 1093 & 1097 & +0.3 & 823 & 823 & 0 & 834 & 827 & -0.9 \\
\hline
\end{tabular}

a Those values enclosed in parentheses are fundamentais shifted by Fermi resonance.

method to a five atom tetrahedral molecule have been given $^{5,6}$ previously and will not be repeated here. The $\mathrm{B}-\mathrm{H}$ bond length in the borohydride ion is not known with a high degree of certainty but it is certainly longer than the $1.19 \mathrm{~A}$ value found ${ }^{7}$ in $\mathrm{BH}_{3} \mathrm{CO}$ since the bond in the latter molecule retains an appreciable amount of $s p^{2}$ character. Price ${ }^{8}$ has suggested $1.26 \mathrm{~A}$ for $\mathrm{NaBH}_{4}$ from empirical relationships between vibrational frequency and internuclear distance, while Ford and Richards ${ }^{9}$ have calculated a distance of $1.255 \mathrm{~A}$ from measurements involving line breadths in nuclear magnetic resonance spectra of the same compound. The value of $1.25 \mathrm{~A}$ used in the calculations thus probably is not seriously in error, and in any case the force constants are not particularly sensitive functions of this parameter.

The potential function in terms of the symmetry coordinates employed ${ }^{6}$ can be written as

$$
2 V=F_{1} S_{1}{ }^{2}+r^{2} F_{2} S_{2}{ }^{2}+F_{3} S_{3}{ }^{2}+r F_{34} S_{3} S_{4}+r^{2} F_{4} S_{4}{ }^{2},
$$

where $S_{1}$ is the nonredundant symmetry coordinate belonging to the $A_{1}$ class, $S_{2}$ the symmetry coordinate of the $E$ class, and $S_{3}$ and $S_{4}$ the stretching and bending coordinates, respectively, of the $F_{2}$ class. Principal force constants are indicated by a single subscript and interaction by a double; $r$ represents the assumed equilibrium value of the internuclear distance. Comparison of the calculated with experimental values of the fundamentals of the four isotopic molecules is made in Table IV. The standard deviation between the two sets (excluding the two $A_{1}$ frequencies involved in Fermi resonances) is $0.5 \%$ with no deviation exceeding $0.9 \%$. Since this agreement probably is better than the uncertainty in the data caused by experimental

${ }^{6}$ A. G. Meister and F. F. Cleveland, Am. J. Phys. 14, 13 (1946).

7 Gordy, Ring, and Burg, Phys. Rev. 78, 512 (1950).

${ }^{8}$ W. C. Price, J. Chem. Phys. 17, 1044 (1949).

P. T. Ford and R. E. Richards, Discussions Faraday Soc. 19, 230 (1955). error and the neglect of anharmonicity, other approaches using more refined potential functions such as the orbital valency type were considered unnecessary. The symmetry force constants yielding the best fit are given in Table $\mathrm{V}$. By employing the defining equations for the symmetry coordinates, these data may be used to calculate values for the more common valence

TABLE V. Force constants for the borohydride ion.

\begin{tabular}{cccc}
\hline \hline $\begin{array}{c}\text { Symmetry force } \\
\text { constant }\end{array}$ & $\begin{array}{c}\text { Value } \\
\left(\mathbf{1 0}^{5} \text { dynes } / \mathrm{cm}\right)\end{array}$ & $\begin{array}{c}\text { Valence force } \\
\text { constant }\end{array}$ & $\begin{array}{c}\text { Value } \\
\left(\mathbf{1 0}^{5} \text { dynes } / \mathrm{cm}\right)\end{array}$ \\
\hline$F_{1}$ & 3.0511 & $k_{r}$ & 2.768 \\
$F_{2}$ & 0.2897 & $k_{r r}$ & 0.094 \\
$F_{3}$ & 2.6737 & $k_{\alpha}-k_{\alpha \alpha}$, & 0.290 \\
$F_{4}$ & 0.2905 & $k_{\alpha}-k_{\alpha \alpha}$, & 0.291 \\
$F_{34}$ & 0.0150 & $k_{r \alpha}-k_{r \alpha}{ }^{\prime}$ & 0.011 \\
\hline
\end{tabular}

force constants. However, since seven valence force constants can be defined in the complete quadratic potential function whereas only five symmetry force constants are available, it is not possible to give a unique set of values for all of the valence force constants. One can, of course, introduce two arbitrary but resonable assumptions and so obtain numbers for all the constants, but rather than introduce this artificiality, the unresolved combinations of valence force constants have been listed as such in Table V. The notation employed is largely self-explanatory, the primed constants being used for interactions between coordinates having only one atom in common.

\section{ACKNOWLEDGMENTS}

This work was supported in part by the U. S. Air Force under Contract AF 33(616)-3343, the sponsoring agency being the Aeronautical Research Laboratory of the Wright Air Development Center, Air Research and Development Command. One of us (A.R.E.) wishes to acknowledge the support given by fellowships awarded by The Procter and Gamble Company and E. I. DuPont de Nemours and Company. 